\title{
Vitamin C-induced Hemolysis: Meta-summary and Review of Literature
}

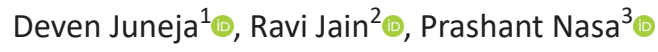

\begin{abstract}
Vitamin C is increasingly being used, and even high doses are considered safe. However, complications including hemolysis have been reported. We performed a systematic search from PubMed, Science Direct, and Google Scholar databases from January 1975 till July 31, 2021. Search terms used were "Vitamin C" OR "ascorbic acid" AND "haemolysis" OR "haemolytic anaemia." Data regarding patient's demographics, outcomes and dose, duration, and form of vitamin C were extracted. Fourteen case reports matched the selected criteria, with age ranging from 3 weeks to 75 years with $78.6 \%$ being males. About $71.4 \%$ were diagnosed to have glucose-6-phosphate dehydrogenase (G6PD) deficiency but previous hemolysis was reported in only two patients, and $57.1 \%$ were prescribed vitamin C for nutritional supplementation. The dose ranged from 1 to $200 \mathrm{~g} /$ day with $57.1 \%$ receiving intravenous formulations. Half of these patients developed other complications including acute kidney injury (AKI), disseminated intravascular coagulation, oxalosis, and methemoglobinemia. About $78.6 \%$ developed complications within 3 days of starting vitamin $C$ and only one death was reported. Vitamin $C$ is generally a safe drug but it should be prescribed with caution and only when benefits outweigh the risks. Physicians should be aware of potential complications like severe hemolysis and AKI, especially when using high doses and in G6PD deficiency.
\end{abstract}

Keywords: Ascorbic acid, Hemolysis, Hemolytic anemia, Vitamin C.

Indian Journal of Critical Care Medicine (2022): 10.5005/jp-journals-10071-24111

\section{INTRODUCTION}

Recent years have witnessed the expanding use of high-dose vitamin $C$ in the management of critically ill patients with sepsis, acute respiratory distress syndrome (ARDS), cancer, and even severe acute respiratory syndrome coronavirus 2 (SARS-CoV-2) pneumonia. ${ }^{1-4}$ Vitamin $C$ or ascorbic acid has been explored in the treatment of sepsis and septic shock because of its antioxidant properties, scavenging free radicals, and postulated bacteriostatic effect. ${ }^{1}$ Besides, working as an enzyme cofactor for many important biological reactions, vitamin $C$ has other proposed mechanism of actions including the prevention of mitochondrial dysfunction and septic cardiomyopathy, regulating macrophage function, and preservation of macro- or microvascular function. ${ }^{1,5}$ Moreover, because of its potential anti-inflammatory and immune-enhancing properties, its therapeutic role has expanded well beyond the treatment of scurvy and promoted even in healthy individuals as "immune-booster". ${ }^{5}$ Vitamin $C$ is a water-soluble vitamin and is generally considered safe to use even in higher doses. Although no serious side effects have been reported in trials evaluating the effectiveness of high-dose vitamin C, safety was not the primary endpoint of these trials. Various case reports have reported several side effects with vitamin $C$ ranging from interference with laboratory tests to life-threatening acute kidney injury (AKI). ${ }^{6,7}$ Here, we aimed to evaluate hemolysis associated with the use of high-dose vitamin $C$ reported in various case reports over the past several decades.

\section{Materials and Methods}

We performed a systematic search for this meta-summary from PubMed, Science Direct, and Google Scholar databases from January 1, 1975, till July 31, 2021. The search terms used were
${ }^{1}$ Institute of Critical Care Medicine, Max Super Speciality Hospital, New Delhi, India

${ }^{2}$ Department of Critical Care Medicine, Mahatma Gandhi Medical College and Hospital, Jaipur, Rajasthan, India

${ }^{3}$ Department of Critical Care Medicine, NMC Specialty Hospital, Dubai, United Arab Emirates

Corresponding Author: Prashant Nasa, Department of Critical Care Medicine, NMC Specialty Hospital, Dubai, United Arab Emirates, Phone: +971501425022, e-mail: dr.prashantnasa@hotmail.com

How to cite this article: Juneja D, Jain R, Nasa P. Vitamin C-induced Hemolysis: Meta-summary and Review of Literature. Indian J Crit Care Med 2022;26(2):224-227.

Source of support: Nil

Conflict of interest: None

"Vitamin C" OR "ascorbic acid" AND "hemolysis" OR "hemolytic anemia." The inclusion criteria were (1) published case reports or case series with individual patient details and (2) a documented history of recent vitamin C use and objective evidence of acute hemolysis related to it. Further, it was filtered for the literature published in the English language and humans. The authors screened all the search results to include only the relevant literature. Duplicate articles from different search databases were excluded. All the authors further reviewed eligible studies for final inclusion based on the availability of individual patient data (Flowchart 1).

All the case reports and case series were evaluated, and the data were extracted for the patient's demographics, dose, duration, formulation of vitamin C therapy, and patient outcomes. A datasheet for evaluation was further prepared for descriptive analysis.

o The Author(s). 2022 Open Access This article is distributed under the terms of the Creative Commons Attribution 4.0 International License (https://creativecommons. org/licenses/by-nc/4.0/), which permits unrestricted use, distribution, and non-commercial reproduction in any medium, provided you give appropriate credit to the original author(s) and the source, provide a link to the Creative Commons license, and indicate if changes were made. The Creative Commons Public Domain Dedication waiver (http://creativecommons.org/publicdomain/zero/1.0/) applies to the data made available in this article, unless otherwise stated. 
Flowchart 1: PRISMA flow diagram of the selected literature for the meta-summary

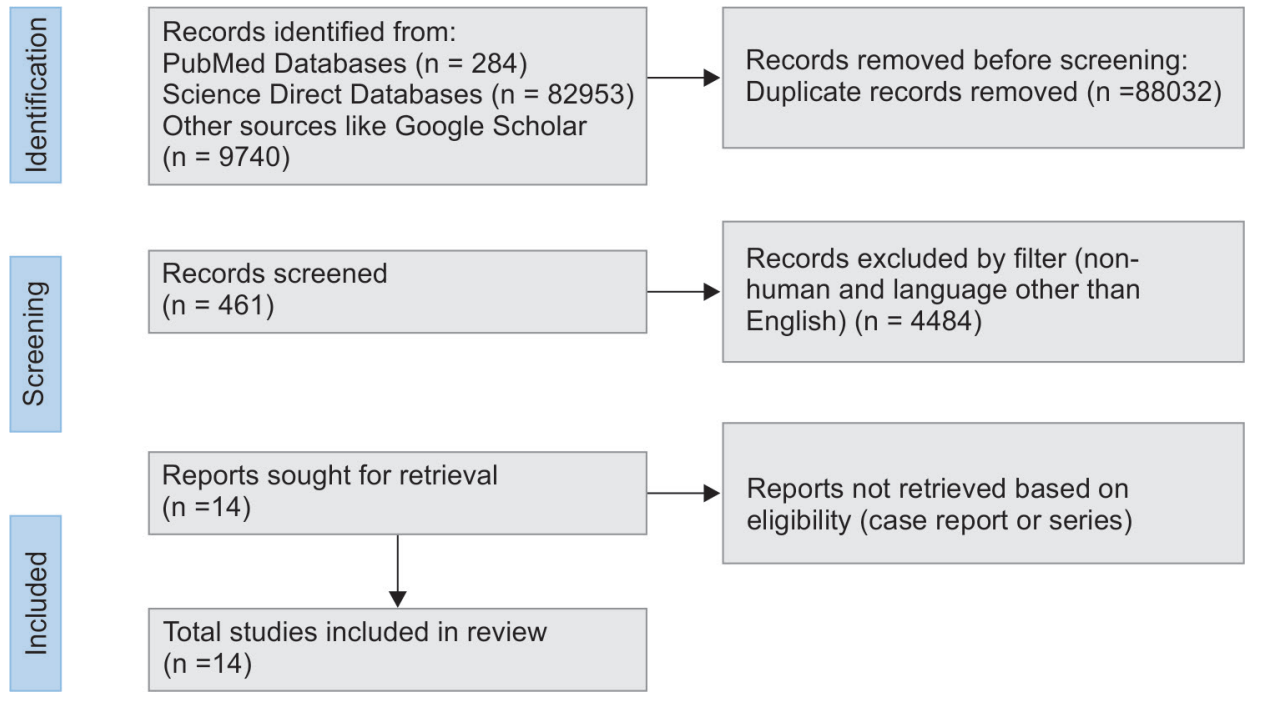

\section{Results}

A total of 14 case reports matched the selected criteria, with publications ranging from 1975 to 2020. Half of the publications were from the Asian Continent. The age of the patients ranged from 3 weeks to 75 years with male predomination $(78.6 \%, 11 / 14)$. About 71.4\% (10/14) were diagnosed with glucose-6-phosphate dehydrogenase (G6PD) deficiency, and in two reports, G6PD deficiency was not reported. A previous history of hemolysis was reported in only two patients (14.3\%). The main indication of prescribing vitamin C was nutritional supplementation in $57.1 \%$ of patients. Other indications included sepsis (two patients), cancer therapy (two patients), treatment of rheumatoid arthritis, and hemifacial spasm (one patient each) (Table 1). ${ }^{8-20}$

The dose of vitamin $C$ received by these patients ranged from $1 \mathrm{~g} /$ day up to $200 \mathrm{~g} /$ day. The 3-week-old infant received a dose of $30 \mathrm{mg} /$ day orally. About $57.1 \%$ of patients received intravenous formulations of vitamin C, but a substantial number (42.9\%) also developed hemolysis after receiving oral formulation. Half of these patients also developed other complications, including $\mathrm{AKI}$, disseminated intravascular coagulation (DIC), oxalosis, and methemoglobinemia, with AKI being the most common associated complication found in $42.9 \%$ of cases. A great majority, $78.6 \%$ (11/14), of patients developed these hemolytic complications within 3 days of starting the vitamin $C$ therapy. Only one death (7\%) was reported in these case reports, but there was a significant associated morbidity (Table 1$)^{8-20}$

\section{Discussion}

The present meta-summary showed that hemolysis might be precipitated after the administration of high-dose intravenous vitamin C, especially in the presence of G6PD deficiency. Oral vitamin $C$, if consumed in large doses for a prolonged period, may also cause hemolysis. Males are generally affected, which may be attributed to a higher incidence of G6PD deficiency. All age groups may be affected, and most of the cases occur within 72 hours of the initiation of therapy. AKI is a commonly associated complication along with acute hemolysis. Deposition of heme pigment in the renal tubules leads to cell injury and death, causing AKI. Even though most of the patients were discharged alive, there was a significant associated morbidity, and rarely, it could be fatal.

Vitamin $C$ is increasingly used for varied clinical conditions. High doses of intravenous vitamin $C$ have been evaluated, with doses up to $100 \mathrm{~g} /$ day used to manage critically ill patients. ${ }^{1}$ The reported side effects are rare and generally mild. These include interference with laboratory tests, lethargy/fatigue, phlebitis, renal stones, hyperglycemia, hypernatremia, muscle cramps, nausea, vomiting, headache, altered mental status, syncope, hemolysis, and AKI. ${ }^{6,7}$ However, specific patient populations like those with underlying renal dysfunction and G6PD deficiency may be at higher risk of developing complications. The polypharmacy in critically ill patients and popular belief on the benign nature of vitamin C may have caused under-recognition and under-reporting of side effects.

G6PD deficiency is the most prevalent enzyme deficiency, with almost $5 \%$ of the world population (more than 329 million) affected. As it is an X-linked recessive disorder, males are generally affected and may develop severe clinical consequences. Most of the patients remain asymptomatic. Hemolytic anemia, secondary to infections or exposure to oxidizing agents/medications, is the most common clinical presentation in adults. ${ }^{21}$ The incidence of G6PD deficiency varies in different regions of the world and different ethnicities. African Americans and Asians are more likely to have G6PD deficiency, and the most widely affected regions of the world include Africa, the Middle East, and Asia. ${ }^{21}$

Vitamin $C$ is considered an antioxidant and has been used to manage hemolysis associated with oxidative damage in patients with G6PD deficiency. ${ }^{22}$ However, it is postulated that it may lead to oxidative stress and hemolysis when used in higher doses. Ascorbic acid is converted in the tissues into its oxidized form, dehydroascorbic acid, which may diminish the intraerythrocyte glutathione stores, especially when present in high concentrations as may be the case when high doses of vitamin $C$ are prescribed. In G6PD deficiency, these glutathione stores are not replenished, leading to oxidative damage and resultant hemolysis. ${ }^{23}$

These side effects have been generally associated with using high doses of vitamin C. However, "high dose" is not clearly defined and is arbitrarily considered a dose of more than $10 \mathrm{~g} /$ day in adults. ${ }^{24}$ Although most of the cases in the present meta-summary had taken 


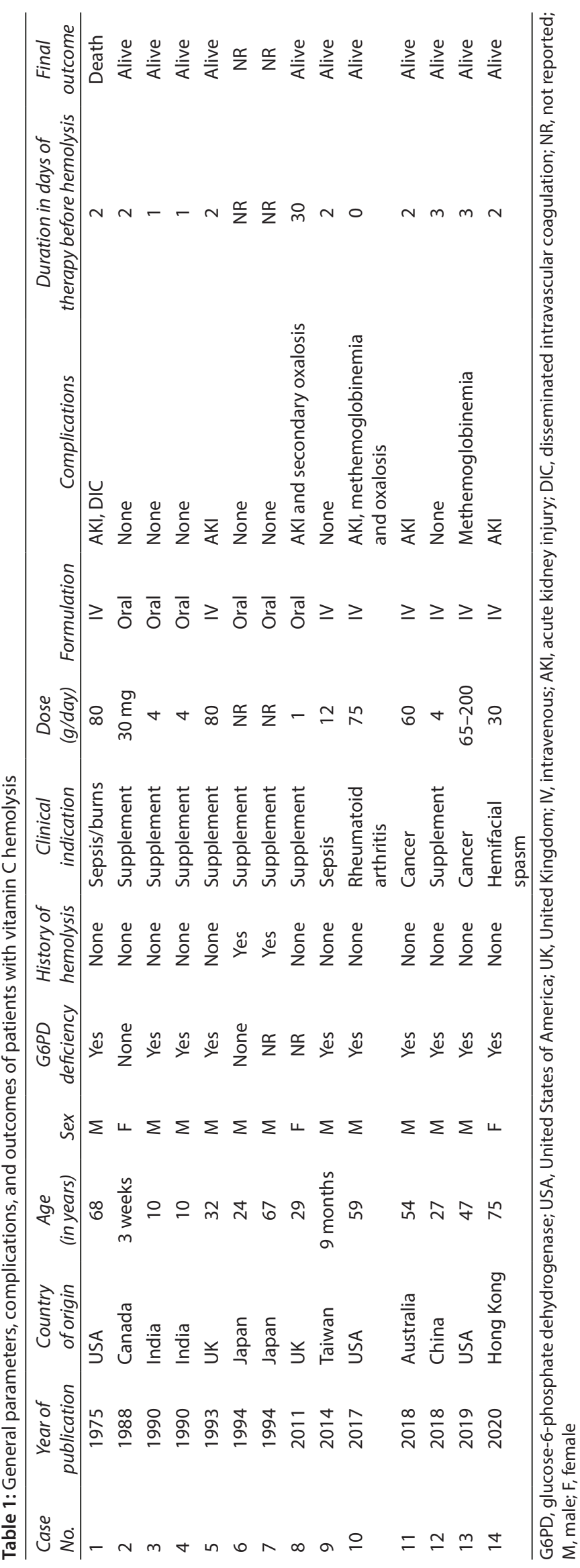

"high dose" of intravenous vitamin $C_{,}^{8,11,15-17}$ we also found cases reported after prolonged low-dose oral vitamin $C_{,}^{14}$ and even after low-dose intravenous therapy. ${ }^{9,10,18}$

\section{Strength and Limitations}

This is the first meta-summary evaluating hemolysis associated with vitamin C therapy. We aimed to make the physicians aware of this possible complication, especially when prescribing high-dose vitamin C. This meta-summary encompassed all the case reports published in the last five decades and tried to identify the risk factors associated with this complication. This meta-summary is limited by a small number of case reports available for analysis. In addition, different criteria were used for the diagnosis of hemolysis in case reports and the incidence of side effects could not be ascertained as we had included data from case reports and not trials. The included studies were only case reports, with a considerable reporting bias, missing data, and hence, the results cannot be generalized.

\section{Conclusion}

Vitamin C administration is generally safe, but like any other drug, it should be prescribed with caution and only when benefits outweigh any side effects. Physicians should be aware of potential complications like severe hemolysis and AKI, which may be precipitated when using high doses of vitamin C, especially in patients with G6PD deficiency. These complications generally occur early after the initiation of therapy. Hence, utmost care and monitoring for these complications in the first 72 hours is suggested.

\section{HighLIGHTS}

Use of high-dose vitamin C may lead to complications like hemolysis and AKI, especially in patients with G6PD deficiency. These complications may occur within 72 hours of the initiation of intravenous or oral therapy.

\section{Contributors}

DJ involved in concept, design, literature search, clinical studies, data acquisition, data analysis, statistical analysis, guarantor, manuscript preparation, manuscript editing, and manuscript review; RJ involved in literature search, clinical studies, data acquisition, data analysis, statistical analysis, and manuscript review; PN involved in data analysis, statistical analysis, manuscript editing, and manuscript review.

\section{ORCID}

Deven Juneja @ https://orcid.org/0000-0002-8841-5678

Ravi Jain (1) https://orcid.org/0000-0001-9260-479X

Prashant Nasa @ i https://orcid.org/0000-0003-1948-4060

\section{References}

1. Somagutta MKR, Pormento MKL, Khan MA, Hamdan A, Hange N, Kc M, et al. The efficacy of vitamin C, thiamine, and corticosteroid therapy in adult sepsis patients: a systematic review and meta-analysis. Acute Crit Care 2021;36(3):185-200. DOI: 10.4266/acc.2021.00108.

2. Fowler AA, Truwit JD, Hite RD, Morris PE, DeWilde C, Priday A, et al. Effect of Vitamin $C$ infusion on organ failure and biomarkers of inflammation and vascular injury in patients with sepsis and severe acute respiratory failure: the CITRIS-ALI randomized clinical trial. Journal of the American Medical Association 2019;322(13):12611270. DOI: 10.1001/jama.2019.11825. 
3. Zasowska-Nowak A, Nowak PJ, Ciałkowska-Rysz A. High-dose Vitamin C in advanced-stage cancer patients. Nutrients 2021;13(3):735. DOI: 10.3390/nu13030735.

4. Suna K, Melahat UŞ, Murat Y, Figen ÖE, Ayperi Ö. Effect of high-dose intravenous vitamin $\mathrm{C}$ on prognosis in patients with SARS-CoV-2 pneumonia. Med Clin (Barc) 2021;S0025-7753(21)00252-9. DOI: 10.1016/j.medcli.2021.04.010.

5. Marik PE. Vitamin C for the treatment of sepsis: the scientific rationale. Pharmacol Ther 2018;189:63-70.DOI:10.1016/j.pharmthera.2018.04.007.

6. Padayatty SJ, Sun AY, Chen Q, Espey MG, Drisko J, Levine M. Vitamin C: intravenous use by complementary and alternative medicine practitioners and adverse effects. PloS One 2010;5(7):e11414. DOI: 10.1371/journal.pone.0011414.

7. Yanase F, Fujii T, Naorungroj T, Belletti A, Luethi N, Carr AC, et al. Harm of IV high-dose Vitamin C therapy in adult patients: a scoping review. Crit Care Med 2020;48(7):e620-e628. DOI: 10.1097/ CCM.0000000000004.

8. Campbell GD, Steinberg MH, Bower JD. Letter: ascorbic acid-induced hemolysis in G-6-PD deficiency. Ann Intern Med 1975;82(6):810. DOI: 10.7326/0003-4819-82-6-810_1.

9. Ballin A, Brown EJ, Koren G, Zipursky A. Vitamin C-induced erythrocyte damage in premature infants. J Pediatr 1988;113(1 Pt 1):114-120. DOI: 10.1016/s0022-3476(88)80544-4.

10. Mehta JB, Singhal SB, Mehta BC. Ascorbic-acid-induced haemolysis in G-6-PD deficiency. Lancet 1990;336(8720):944. DOI: 10.1016/01406736(90)92317-b.

11. Rees DC, Kelsey H, Richards JD. Acute haemolysis induced by high dose ascorbic acid in glucose-6-phosphate dehydrogenase deficiency. BMJ 1993;306(6881):841-842. DOI: 10.1136/bmj.306.6881.841.

12. Iwamoto N, Kawaguchi T, Horikawa K, Nagakura S, Hidaka M, Kagimoto T, et al. Haemolysis induced by ascorbic acid in paroxysmal nocturnal haemoglobinuria. Lancet 1994;343(8893):357. DOI: 10.1016/ s0140-6736(94)91195-9.

13. Iwamoto N, Kawaguchi T, Horikawa K, Nagakura S, Hidaka M, Kagimoto T, et al. Haemolysis induced by ascorbic acid in paroxysmal nocturnal haemoglobinuria. Lancet 1994;343(8893):357. DOI: 10.1016/ s0140-6736(94)91195-9.

14. Stepien KM, Prinsloo $P$, Hitch T, McCulloch TA, Sims R. Acute renal failure, microangiopathic haemolytic anemia, and secondary oxalosis in a young female patient. Int J Nephrol 2011;2011:679160. DOI: 10.4061/2011/679160.

15. Huang Y-C, Chang T-K, Fu Y-C, Jan S-L. C for colored urine: acute hemolysis induced by high-dose ascorbic acid. Clin Toxicol Phila Pa 2014;52(9):984. DOI: 10.3109/15563650.2014.954124.

16. Quinn J, Gerber B, Fouche R, Kenyon K, Blom Z, Muthukanagaraj P. Effect of high-dose Vitamin C infusion in a glucose-6-phosphate dehydrogenase-deficient patient. Case Rep Med 2017;2017:5202606. DOI: 10.1155/2017/5202606.

17. Rees MJ, Strach MC, Burbury K, Phillips K. Massive oxidative haemolysis and renal failure caused by high dose vitamin C. Med J Aust 2018;209(6):248-249. DOI: 10.5694/mja17.00998.

18. Wu S, Wu G, Wu H. Hemolytic jaundice induced by pharmacological dose ascorbic acid in glucose-6-phosphate dehydrogenase deficiency: a case report. Medicine 2018;97(51):e13588. DOI: 10.1097/ MD. 0000000000013588.

19. Nelson R, Ma J, Latifi N, Sell R. Intravascular hemolysis and methemoglobinemia from high dose vitamin C. Eur J Med Case Rep 2020;4(2):49-51. DOI: 10.24911/ejmcr/ 173-1544044604.

20. Lo YH, Mok KL. High dose vitamin C induced methemoglobinemia and hemolytic anemia in glucose-6-phosphate dehydrogenase deficiency. Am J Emerg Med 2020;38(11):2488.e3-2488.e5. DOI: 10.1016/j.ajem.2020.05.099.

21. Nkhoma ET, Poole C, Vannappagari V, Hall SA, Beutler E. The global prevalence of glucose-6-phosphate dehydrogenase deficiency: a systematic review and meta-analysis. Blood Cells Mol Dis 2009;42(3):267-278. DOI: 10.1016/j.bcmd.2008.12.005.

22. Krishna AP, Sivakumar T, Jin C, Li S-H, Weng Y-J, Yin J, et al. Antioxidant and hemolysis protective effects of polyphenol-rich extract from mulberry fruits. Pharmacogn Mag 2018;14(53):103. DOI: 10.4103/ pm.pm_491_16.

23. Zhang ZZ, Lee EE, Sudderth J, Yue Y, Zia A, Glass D, et al. Glutathione depletion, pentose phosphate pathway activation, and hemolysis in erythrocytes protecting cancer cells from Vitamin C-induced oxidative stress. J Biol Chem 2016;291(44):22861-22867. DOI: 10.1074/ jbc.C116.748848.

24. Wang $Y$, Lin H, Lin B, Lin J. Effects of different ascorbic acid doses on the mortality of critically ill patients: a meta-analysis. Ann Intensive Care 2019;9(1):58. DOI: 10.1186/s13613-019-0532-9. 